DOI: https://doi.org/10.11144/Javeriana.upsy16-4.ecvf

\title{
Escala de Calidad de Vida Familiar: validación y análisis en población chilena
}

Family Quality of Life Scale (FQLS): validation and analysis in a Chilean population

Recepción: 07 Junio 2016 | Aprobación: 31 Marzo 2017

\author{
Natalia Bello-Escamilla \\ Universidad del Bío-Bío, Chile \\ JOUCELYN RIVADENEIRA ${ }^{\text {a }}$ \\ Universidad del Bío-Bío, Chile \\ ORCID: http://orcid.org/0000-0001-6917-1690 \\ Marcela Concha-Toro \\ Universidad del Bío-Bío, Chile \\ Ariel Soto-Caro \\ Universidad de Concepción, Chile \\ Ximena Díaz-Martínez \\ Universidad del Bío-Bío, Chile
}

a Autor de correspondencia. Correo electrónico: jrivadeneira@ubiobio.cl

Para citar este artículo: Bello-Escamilla, N., Rivadeneira, J., Concha-Toro, M., Soto-Caro, A., \& Díaz-Martínez, X. (2017). Escala de Calidad de Vida Familiar: validación y análisis en población chilena. Universitas Psychologica, 16(4), 1-10. https://doi.org/1 0.11144/Javeriana.upsy16-4.ecvf

\section{RESUMEN}

El propósito de este estudio es presentar la validación de la Escala de Calidad de Vida Familiar en población chilena y analizar las dimensiones de este instrumento a través del mapa de área crítica. Se utilizó un estudio transversal, correlacional y causal en 316 familias. Se aplicó la versión española de la Escala de Calidad de Vida Familiar. Se condujo un análisis factorial exploratorio y confirmatorio. Los resultados indicaron que el alpha de Cronbach para la categoría de Importancia fue de 0.884 y para Satisfacción fue de 0.891. El análisis factorial exploratorio encontró cinco dimensiones para ambas categorías, y el análisis factorial confirmatorio mostró cargas mayores a 0.5 para cada dimensión. Respecto a la validez discriminante, las cargas cruzadas se movieron entre 0.64 a 0.97 para Importancia y 0.62 a 0.98 para Satisfacción. En cuanto a la validez convergente, cada dimensión alcanzó una varianza extraída sobre 0.5. Las conclusiones del estudio indican que el instrumento resultó ser confiable y válido para medir aspectos de la calidad de vida familiar en población chilena. Posee altos niveles de consistencia interna y una adecuada validez discriminante y convergente.

Palabras clave

calidad de vida; familia; estudios de validación.

\section{ABSTRACT}

The aim of this research is to validate the Family Quality of Life Scale (FQLS) in a Chilean population and to analyze the factor structure (scope) of this research tool through the critical area map. Crosssectional, correlational, and causal study was carry on 316 families. The Spanish version of the FQSL was used. An exploratory and confirmatory factor analysis was conducted. Results showed Cronbach's alpha for the FQLS subscales on Importance rate was 0.884 and on Satisfaction 0.891. The exploratory factor analysis found five dimensions for both 
categories, and the confirmatory factor analysis showed factor loadings greater than 0.5 for each dimension. Regarding discriminant validity, the cross-loadings varied between 0.64 to 0.97 on Importance and 0.62 to 0.98 on Satisfaction. In relation to convergent validity, each dimension reached a variance above 0.5 . Conclusions of the study proved a reliable and valid tool to measure features of family quality of life in a Chilean population. The instrument has high levels of internal consistency and suitable discriminant and convergent validity.

Keywords

quality of life; family; validation studies.

La familia es el pilar fundamental del desarrollo humano y unidad principal de toda sociedad. Como institución tiene una incidencia decisiva en la configuración de la calidad de vida (CV) de sus miembros, fundamentalmente en la de los niños y niñas. La calidad de vida familiar (CVF) puede entenderse como un estado dinámico de bienestar de la familia, definido de forma colectiva y subjetiva y valorado por sus miembros, en el que interactúan las necesidades a nivel individual y familiar (Zuna, Turnbull, \& Summers, 2009). Se entiende por tanto que la CV centrada en la familia está estrechamente vinculada con la CV centrada en la persona, siendo además influenciada significativamente por factores personales y socioculturales (Schalock \& Verdugo, 2007).

La fundamentación, consenso y avances vinculados a la CV individual son más amplios que los logrados respecto a la CVF (Sainz \& Verdugo, 2006), pues, a juicio de estos autores, la dificultad que existe para evaluar la CVF radica en los componentes subjetivos y objetivos del concepto, los cuales se mueven entre la Importancia y la Satisfacción que la propia familia otorga a aspectos de su vida y aquellos que pueden ser directamente medibles u observables.

Una forma de entender y medir la CVF es considerar que se trata de un constructo social multidimensional, compuesto de dimensiones dinámicas e independientes (Samuel, Rillota, \& Brown, 2012). Bajo esta premisa, en las últimas décadas se han producido importantes avances relacionados con la valoración de la
CVF, especialmente en Estados Unidos y en menor medida en Latinoamérica.

Por otra parte, es importante reconocer que habitualmente el interés de los estudios se ha centrado en dos aspectos diferenciados: por un lado, se ha profundizado en las consecuencias de la discapacidad de un miembro de la familia en la CVF (Aya \& Córdoba, 2013; Córdoba, Gómez, \& Verdugo, 2008; Fernández-Faúndez, Arias, Gómez, \& Jorrín, 2012; Giné et al., 2015; González-del-Yerro, Simón, Cagigal, \& Blas, 2013; Muñoz, Poblete, \& Jiménez, 2012; Rodríguez, Verdugo, \& Sánchez, 2008) y por otro, se han abordado las repercusiones que algunas condiciones médicas pudiesen tener en la CVF (Borth-Bruhns, Seitz, Besier, West, \& Goldbeck, 2009; Goldbeck et al., 2005; Loga et al., 2012). Como contraparte, es escaso el conocimiento que se aprecia en torno a la CVF en otros ámbitos, por lo que en la actualidad se evidencia un vacío empírico que urge asumir a la comunidad científica. En Chile, la situación no es diferente, dado que existen escasos estudios en torno a la CVF (Muñoz et al., 2012; Navarrete \& Ossa, 2013; Ossa, Navarrete, \& Jiménez, 2014), y en la actualidad no se cuenta con instrumentos validados en población chilena.

La relevancia de evaluar la CVF radica en que es necesario avanzar en el conocimiento en torno a la familia. Si bien es cierto, hay estudios de este núcleo de la sociedad, conocer cómo perciben sus condiciones de vida puede transformarse en una herramienta útil tanto para los programas que se abocan al trabajo directo como para otras instancias dirigidas al fortalecimiento de la familia como institución en la cual se llevan a cabo los principales y más importantes aprendizajes humanos. Vasta experiencia en esta temática ha demostrado tener el Beach Center on Disability (2003) en Estados Unidos, institución abocada a la investigación y asesoría a las personas con discapacidad y sus familias. Dentro de sus logros más importantes aparece la creación de la Escala de Calidad de Vida Familiar, la cual se elabora con el propósito de conocer dos dimensiones de la calidad de vida: la Importancia que las familias otorgan a ciertos aspectos del contexto social y familiar 
que les hacen tener una buena vida juntos y por otra parte, la Satisfacción que pueden llegar a tener respecto a estos mismos aspectos. Al respecto, los autores señalan que se consideró la dimensión Importancia debido no solo a que se trata de aspectos relevantes asociados a la CVF, sino porque representa un constructo estable, ya que tiende a variar en menor medida a lo largo del tiempo. En cuanto a la Satisfacción, los autores sostienen que, si bien se trata de un constructo menos estable, permite obtener información sobre el estado de cumplimiento de dichos aspectos que para la familia son relevantes en relación a la CV (Park et al., 2003). Se trata de una escala que ha sido validada en población española (Sainz \& Verdugo, 2006) y colombiana (Verdugo, Córdoba, \& Gómez, 2005) con lo cual se ha logrado extender el conocimiento respecto a la CVF fuera del contexto de los Estados Unidos.

Continuando esta línea investigativa, el primer objetivo que se plantea el presente estudio es asumir el vacío empírico que existe actualmente en Chile, mediante la validación de la Escala de Calidad de Vida Familiar (ECVF), contribuyendo esto tanto a la profundización y entendimiento de la familia desde un punto de vista holístico e integrador, así como para orientar las intervenciones de los equipos multi e interdisciplinarios que trabajan con familias y fortalecer la labor investigativa. El segundo objetivo de esta investigación busca analizar las dimensiones del instrumento en la población estudiada a través del mapa del área crítica de la calidad de vida familiar.

\section{Método}

\section{Participantes}

Estudio de tipo transversal, correlacional y causal con 316 madres, padres o adultos responsables de niños y niñas pertenecientes a establecimientos educacionales de administración municipal de la ciudad de Chillán (Chile).

De acuerdo al Sistema Nacional de Información Municipal de Chile para los años
2014 y 2015 en la ciudad de Chillán existen aproximadamente 1535 alumnos de enseñanza prebásica y de primer año de educación básica. Para dicha población se requirieron 308 observaciones que permitieron construir una muestra con $5 \%$ de error, $95 \%$ de confianza y $50 \%$ de distribución de la muestra. Con las 316 observaciones recogidas se obtiene un error del $4.91 \%$.

Los participantes estuvieron conformados en un $82.98 \%$ por padres/madres y en un $17.02 \%$ por tíos, abuelos u otros adultos responsables. El $22.3 \%$ de los sujetos no tiene educación formal, el $31.4 \%$ posee enseñanza básica, el $37.8 \%$ tiene enseñanza media y un $8.5 \%$ educación universitaria.

Con respecto al ingreso económico mensual del grupo familiar, el $52.66 \%$ de la muestra posee un ingreso familiar por debajo de los $\$ 182.793$. El $42.55 \%$, un ingreso familiar mensual que se ubica entre los $\$ 182.793$ y $\$ 333.909$; en tanto que el $10.64 \%$ refiere percibir uno mayor a los $\$ 333.910$. Un $4.79 \%$ no sabe o no contesta.

\section{Procedimiento}

Este estudio forma parte de un proyecto regular financiado por la Universidad del Bío-Bío, cuyo objetivo se centró en la evaluación de aspectos del estado físico y nutricional de niños y niñas entre 5 y 7 años de edad. Las familias fueron contactadas a través de los establecimientos educacionales a los cuales asistían los/as niños y niñas, solicitando el consentimiento firmado de madre, padre o adulto responsable previo a su incorporación en la investigación. Este estudio fue revisado y aprobado por el Comité de Ética de la Universidad del Bío-Bío.

\section{Instrumento}

Se aplicó la ECVF desarrollada originalmente por el Beach Center de la Universidad de Kansas en Estados Unidos en el 2003 (Farran, Lederberg, \& Jackson, 2009), dirigida a evaluar la CVF en familias con hijos e hijas con discapacidad. Esta escala ha sido traducida y adaptada a 
población española por Sainz y Verdugo (2006), versión en la cual se basa esta validación. La escala valora dos categorías: por una parte, la Satisfacción que experimentan las familias frente a cada uno de los indicadores de CVF, y por otra parte, la Importancia que conceden a dichos indicadores. De esta forma, se debe responder a ambas categorías, es decir, qué tan importante es un indicador para la persona, así como la Satisfacción actual con dicho indicador.

El instrumento es una escala tipo Likert, de 25 ítems con cinco categorías, que van desde muy insatisfecho (1) hasta muy satisfecho (5), y desde poco importante (1) hasta importantísimo (5). Presenta cinco indicadores de calidad de vida: interacción familiar, papel de los padres, bienestar emocional, y bienestar físico y material. La última dimensión es específica y está dirigida solo a aquellas familias que cuentan con una persona con discapacidad en el mismo hogar, y se denomina recursos y apoyo relacionado con la discapacidad.

La Interacción familiar se refiere a la relación que tienen los miembros de la familia entre sí, y el clima emocional que caracteriza dicha relación. Incluye aspectos como ambiente interaccional, comunicación, apoyo mutuo y flexibilidad en la planificación. El Papel de los padres es el factor que evalúa la forma en que los adultos proveen orientación o guía, disciplina y enseñanza a los niños y adolescentes de la familia. El factor Bienestar emocional se refiere a la salud física y mental de la familia, y las condiciones de los contextos físicos dentro de los cuales viven los miembros de la familia (hogar, colegio, trabajo, vecindario y comunidad). El Bienestar físico y material mide aspectos como el cuidado familiar, actividades diarias del hogar, obtención de ayuda externa, habilidades y oportunidades para tener relaciones con personas ajenas a la familia, contar con ingresos que le permitan a la familia por lo menos cubrir los gastos, identidad, respeto, reducción del estrés y libre elección. Los Recursos y apoyo relacionado con la discapacidad se refiere a las oportunidades con que cuenta el miembro de la familia con discapacidad para participar en diferentes actividades tales como educación, trabajo, tiempo libre. Además, las actividades que desarrollan los miembros de la familia para apoyarse mutuamente u obtener apoyo de otros.

La puntuación total del instrumento se obtiene de la suma de las puntuaciones. La puntuación mínima es de 25 puntos y la máxima de 125 puntos. Una puntuación más alta indica un mejor nivel de calidad de vida, tanto para Importancia como para Satisfacción.

\section{Análisis}

Se realizaron análisis de fiabilidad por ítem, constructo y de escala. Además, se revisó la validez convergente y cruzada. Finalmente, se interpretaron las respuestas medias y el mapa del área crítica de la CVF. Todo esto utilizando el software SPSS versión 19.

\section{Resultados}

\section{Fiabilidad}

La fiabilidad individual de cada ítem se comprobó a través de las cargas cruzadas, o correlaciones simples, sobre 0.707 (Carmines \& Zeller, 1979), así más del $50 \%$ de la varianza del parámetro es compartida con la variable (Bollen, 1989). La fiabilidad de cada constructo se comprobó a través de los valores alpha de Cronbach superiores a 0.7 (Bagozzi \& Yi, 1988) y la fiabilidad compuesta sobre 0.7 (Tabla 1).

\section{TABLA 1}

Análisis de fiabilidad

\begin{tabular}{lcccc}
\hline & \multicolumn{2}{c}{ Fiabilidad compuesta } & \multicolumn{2}{c}{ alpha-Cronbach } \\
\cline { 2 - 5 } & Importancia & Satisfacción & Impotancia & Satisfaceín \\
\hline Bienestar emocional & 0.8707 & 0.8553 & 0.7994 & 0.7675 \\
Bienestar fisico y material & 0.7847 & 0.785 & 0.593 & 0.593 \\
Interacción familiar & 0.8496 & 0.8532 & 0.7797 & 0.7936 \\
Papel de los padres & 0.8741 & 0.8599 & 0.8214 & 0.8061 \\
Recursos $y$ apoyo & 0.9907 & 0.99 & 0.9875 & 0.9899
\end{tabular}

Fuente: elaboración propia.

Para la consistencia interna del instrumento se obtuvieron los alpha de Cronbach para Importancia y Satisfacción con valores sobre 0.8 cada uno: 0.884 y 0.891 , respectivamente. 


\section{Validez}

El análisis factorial es ampliamente utilizado en la literatura para validar encuestas de orden subjetivo. En este caso se implementó un Análisis Factorial Exploratorio (AFE, en adelante) para detectar las dimensiones latentes de la encuesta y un Análisis Factorial Confirmatorio (AFC, en adelante) para evaluar las cargas. Para ello se utilizó el método de Componentes Principales sobre la matriz de correlaciones y VARIMAX como método de rotación. Tanto para Importancia como para Satisfacción, el AFE encontró cinco dimensiones, y el AFC mostró cargas mayores a 0.5 para cada dimensión, indicando su relevancia en la medida de cada categoría y cada dimensión. Con respecto a la validez discriminante, las cargas cruzadas se movieron entre 0.64 a 0.97 para Importancia y 0.62 a 0.98 para Satisfacción, asegurando que cada constructo difiera del resto, dentro del instrumento.

Con respecto a la validez convergente, cada dimensión alcanzó una varianza extraída (AVE) sobre 0.5 (Fornell \& Larcker, 1981), la que refleja la cantidad total de la varianza de los indicadores recogida por el constructo latente. Cuanto mayor sea su valor, más representativos son los indicadores de la dimensión crítica en la que cargan. Todos los factores exceden el valor recomendado de 0.5 (Bagozzi \& Yi, 1988; Hair, Anderson, Tatham, \& Black, 2009) (Tabla 2).

TABLA 2

Análisis de validez

\begin{tabular}{lcc}
\hline & \multicolumn{2}{c}{ AVE } \\
\cline { 2 - 3 } & Importancia & Satisfacción \\
\hline Bienestar emocional & 0.6301 & 0.6034 \\
Bienestar fisico y material & 0.5503 & 0.5505 \\
Interacción familiar & 0.5309 & 0.4929 \\
Papel de los padres & 0.5834 & 0.514 \\
Recursos y apoyo & 0.9638 & 0.9707 \\
\hline
\end{tabular}

Fuente: elaboración propia.

Complementario a la aplicación de la Escala, se elaboró el mapa del área crítica de la CVF, el cual estructura gráficamente las intersecciones posibles entre Importancia y Satisfacción, identificándose de esta forma los indicadores de cada una de las dimensiones de la CVF que estuvieran ubicados en el área crítica (Córdoba et al., 2008).

Como se aprecia en la Tabla 3, en el cuadrante 1, un $8.64 \%$ del total de las familias piensa que es importantísimo que "Mi familia cuente con servicio dental cada vez que lo necesita" (ítem 20), sin embargo, se encuentran muy insatisfechas con este ítem, puesto que no cuentan con atención dental oportuna. Asimismo, un $3.18 \%$ piensa que es importantísimo que "Mi familia cuente con servicio médico cuando lo necesita" (ítem 15) y un $2.73 \%$ que "Mi familia se sienta segura en la casa, en el trabajo y en el barrio" (ítem 21), no obstante, las familias se encuentran muy insatisfechas con el cumplimiento de estos ítems. Todos pertenecientes a la dimensión Bienestar físico y material.

TABLA 3

Mapa del área crítica de la Calidad de Vida Familiar

\begin{tabular}{cccccc}
\hline & Cuadrante 3 & \multicolumn{5}{c}{ Cuadrante 4 } \\
\hline Pregunta & Cantidad & $\%$ & Pregunta & Cantidad & $\%$ \\
\hline 20 & 3 & 1.36 & 2 & 5 & 2.27 \\
9 & 2 & 0.91 & 13 & 4 & 1.82 \\
21 & 2 & 0.91 & 16 & 4 & 1.82 \\
\hline Pregunta & Cantidad & $\%$ & Pregunta & Cantidad & $\%$ \\
\hline 20 & 19 & 8.64 & 20 & 20 & 9.09 \\
15 & 7 & 3.18 & 6 & 16 & 7.27 \\
21 & 6 & 2.73 & 15 & 9 & 4.09 \\
\hline
\end{tabular}

En el cuadrante 2 se observan, al igual que en el cuadrante 1 , los ítems 20 y 15, en los cuales un $9.09 \%$ y $4.09 \%$ de las familias, respectivamente, considera que es importantísimo el cumplimiento de ambos ítems, pero que, sin embargo, se encuentran insatisfechos con su consecución. Lo mismo, con el ítem 6 "Los miembros de mi familia cuenten con transporte para llegar a los lugares que necesitan", donde un $7.27 \%$ del total de las familias piensa que es importantísimo 
el cumplimiento de este ítem, sin embargo, se encuentran insatisfechos, ya que no cuentan siempre con transporte para trasladarse. Todos ítems pertenecientes a la dimensión Bienestar físico y material.

En el cuadrante 3, nuevamente se advierten los indicadores 20 y 21 con un $1.36 \%$ y $0.91 \%$, respectivamente. Adicionalmente, se distingue el ítem 9 "Los miembros de mi familia tengan el tiempo para desarrollar sus propios intereses" de la dimensión Bienestar emocional, donde un 0.91 $\%$ del total de las familias cree que es un ítem muy importante, sin embargo, se encuentran muy insatisfechas con su cumplimiento.

Por último, en el cuadrante 4 , se observa que un $2.27 \%$ de las familias piensan que es muy importante que "Los miembros de mi familia ayuden a los/as niños/as a ser independientes" (ítem 2), sin embargo, se encuentran insatisfechos con el cumplimiento de este ítem de la dimensión Papel de los padres. Asimismo un $1.82 \%$ de las familias piensa que es muy importante que "Mi familia cuente con ayuda externa disponible para ayudar con las necesidades particulares de todos los miembros de la familia" (ítem 13) y que "Mi familia encuentre la manera de cubrir nuestros gastos" (ítem 16), no obstante, se encuentran insatisfechos con la realización de ambos ítems pertenecientes a las dimensiones de Bienestar emocional y Bienestar físico y material, respectivamente.

En estas familias, las necesidades prioritarias (indicadores ubicados en los cuadrantes 1 y 2 del área crítica) pertenecen a la dimensión de Bienestar físico y material. Estas necesidades deben jerarquizarse con el propósito de buscar estrategias y acciones que permitan convertir los puntos fuertes en oportunidades para superar los puntos débiles que constituyen amenazas para la calidad de vida familiar (Córdoba, Verdugo, \& Gómez, 2011).

Con respecto a cada factor, las respuestas medias más bajas se encontraron en la Satisfacción de Bienestar emocional e Importancia de Bienestar físico y material (3.67), mientras que la más alta la posee la
Importancia de Recursos y apoyo relacionado con la discapacidad (4.51).

Analizando por ítem, con respecto a Importancia, el ítem "Mi familia cuente con ayuda externa disponible para ayudar con las necesidades particulares de todos los miembros de la familia" posee la respuesta media más baja (3.65) y "Mi familia disfrute de estar junta" la más alta (4.74). Por otro lado, con respecto a Satisfacción, el ítem "Los miembros de mi familia cuenten con transporte para llegar a los lugares que necesitan" es la respuesta media más baja (3.51) y el ítem "Los miembros de mi familia muestren que se aman y preocupan por los demás" es la más alta (4.39).

Observando la Tabla 4, en general, Importancia declara una respuesta media superior a Satisfacción. Interacción familiar resulta ser en promedio lo más importante, seguido por Recursos y apoyo relacionado con la discapacidad y Bienestar emocional lo menos importante. Recursos y apoyo relacionado con la discapacidad resulta ser donde hay más Satisfacción y Bienestar físico y material donde hay menos.

TABLA 4

Respuesta media por factor y dimensión

\begin{tabular}{ccccccc}
\hline & General & IF & PP & BE & RAD & BFM \\
\hline Importancia & 3.69 & 4.47 & 4.41 & 3.67 & 4.51 & 4.23 \\
Satisfacción & 3.37 & 4.13 & 4.05 & 3.96 & 4.44 & 3.67 \\
\hline
\end{tabular}

Nota. IF $=$ Interacción familiar; PP $=$ Papel

de padres; $\mathrm{BE}=$ Bienestar emocional; RAD

$=$ Recursos y apoyo relacionado con la

discapacidad; BFM = Bienestar físico y material.

Fuente: elaboración propia.

\section{Discusión}

El primer objetivo de este estudio fue validar un instrumento para medir la CVF. Se utilizó la versión española de la ECVF, el que resultó ser un instrumento confiable y válido para medir aspectos de la CVF, en una población chilena típica. Posee altos niveles de consistencia interna y una adecuada validez discriminante y convergente, mostrando resultados consecuentes con las validaciones realizadas en Estados 
Unidos (Hoffman, Marquis, Poston, Summers, \& Turnbull, 2006), Colombia (Córdoba et al., 2008), China (Wang, 2010) y Taiwán (Chiu, 2013). En coincidencia con estos estudios, no surgieron ítems eliminables.

Por otra parte, el instrumento utilizado presenta robustez estadística, dado que una distorsión en la cantidad de factores, pasando de 5 a 7, y eliminando la categoría de Importancia, continúa siendo válido, tal como el ejercicio realizado por Giné et al. (2013) en España.

Los resultados para Importancia, en general, son más altos en todos los indicadores que los obtenidos en Satisfacción, lo que implica que las familias del estudio no están conformes en la actualidad con la percepción de su CVF. La única dimensión que ha sido valorada de manera distinta por la muestra es la de Bienestar emocional, donde el nivel percibido de Satisfacción es mayor que la Importancia que se le otorga. Por lo tanto, si bien por una parte existe una tendencia a no estar satisfechos con la CVF, por otra parte, esta insatifacción general no afecta la percepción subjetiva de sentirse bien y en tranquilidad. En atención a ello, es posible que las familias de la muestra posean otras herramientas o estrategias personales que les permitan afrontar de manera efectiva su situación actual y afrontar con cierto equilibrio emocional las otras condiciones de vida con las que no se está satisfecho.

Si se comparan algunos resultados con otros estudios que han utilizado la escala, es posible encontrar algunas diferencias y similitudes. Por ejemplo, en la investigación efectuada en Colombia (Córdoba et al., 2008), en la categoría Satisfacción es la Interacción Familiar el factor más alto en promedio, mientras que en Chile el mismo factor es el segundo más alto. Con base en ello, es posible afirmar que el clima emocional que se produce entre los miembros de la familia es un factor que interesa en ambos estudios, pudiendo existir aquí un componente cultural destacable en torno al papel central de la familia en la sociedad.

Así también y en atención a las diferencias, la muestra colombiana presenta los niveles más bajos en Recursos y apoyo relacionado con la discapacidad, en tanto en la muestra chilena resultó ser el indicador con el promedio más alto. En efecto, en Chile se han producido una serie de avances en materia de atención a las personas con discapacidad, incluyendo políticas de inclusión y mejoramiento al acceso en las áreas de educación, salud, laboral, entre otras.

En relación con el segundo objetivo de este trabajo que fue analizar la CVF en la población estudiada a través de la matriz diagnóstica, mapa del área crítica, los resultados fueron coincidentes con el estudio de Córdoba et al. (2008), donde el cuadrante 3 posee la menor cantidad de respuestas similares entre los encuestados, mientras que el cuadrante 2 es donde más coincidencias existen. Es decir, en ambos estudios hubo un mayor número de familias que se sienten insatisfechas con el cumplimiento de las dimensiones de la CVF que consideraron importantísimas. Asimismo, fueron menos las familias que concordaron en que aquellas dimensiones estimadas como muy importantes para la CVF se cumplen de manera muy insatisfactoria, de manera que este análisis se torna crucial para priorizar acciones de trabajo grupal con las familias.

En cuanto a las limitaciones del estudio, una de ellas podría deberse a la muestra, la cual no es representativa de entornos rurales ni de otros contextos con mayor diversidad socioeconómica. Así, también, el presente estudio no ha contemplado la inclusión de otros tipos de administración educacional como los colegios subvencionados particulares y particulares- presentes en el país, lo cual podría eventualmente otorgar mayor variabilidad a los resultados. Futuras investigaciones podrían abarcar asimismo a otros miembros de la familia, a fin de poseer una visión más completa de las percepciones en torno a la CVF.

La CVF es un elemento central asociado al desarrollo humano que permite conocer aspectos esenciales en la trayectoria individual y familiar de las personas. El estudio de la CV facilita no solo la comprensión de los complejos procesos que se dan entre los miembros de la familia, sino a su vez, los procesos socializadores que se producen en los diferentes contextos de 
desarrollo y cómo la familia se relaciona con éstos. Avanzar por tanto en el conocimiento de la CVF se convierte en un insumo potencialmente importante en relación con la formulación de políticas públicas y decisiones que involucran el mejoramiento de la CV. En este sentido, disponer de una escala de CVF en el contexto chileno permitirá a los equipos profesionales y técnicos de diferentes ámbitos de actuación, contar con un instrumento que complemente las intervenciones y apoyo dirigido a las familias. Finalmente, esta escala facilita avanzar en el conocimiento científico en torno a la CVF, contribuyendo así a superar el vacío empírico en el contexto nacional y latinoamericano.

\section{Agradecimientos}

Este estudio forma parte del proyecto DIUBB 145523 3/R financiado por la Universidad del Bío-Bío. Los autores agradecen la participación de las familias en la realización de esta investigación.

\section{Referencias}

Aya, V., \& Córdoba, L. (2013). Asumiendo juntos los retos: calidad de vida en familias de jóvenes con discapacidad intelectual. Revista de la Facultad de Medicina, 61(2), 155-156.

Bagozzi, R., \& Yi, Y. (1988). On the evaluation of structural equation models. Journal of the Academy of Marketing Science, 16(1), 74-94. https://doi.org/10.1007/BF02723327

Beach Center on Disability. (2003). Family Quality of Life Scale. Psychometric characteristics and scoring key. Recuperado de http://www.beachcenter.org/resource_li brary/beach_resource_detail_page.aspx?int ResourceID $=2391$

Bollen, K. (1989). A new incremental fit index for general structural equation models. Sociological Methods Eु Research, 17(3), 303-316.

Borth-Bruhns, T., Seitz, D., Besier, T., West, C., \& Goldbeck, L. (2009). And what about the rest of the family? Quality of life and behavioral disorders in families of patients with chronic heart disease prior to and following a family orientated rehabilitation. Clinical Research in Cardiology, 98(9), 595-596.

Carmines, E., \& Zeller, R. (1979). Reliability and validity assessment. Thousand Oaks, CA: Sage.

Chiu, C. (2013). Family needs and family quality of life for Taiwanese families of children with intellectual disability and developmental delay (Tesis doctoral). University of Kansas, Kansas.

Córdoba, L., Gómez, J., \& Verdugo, M. (2008). Calidad de vida familiar en personas con discapacidad: un análisis comparativo. Universitas Psychologica, 7(2), 369-383.

Córdoba, L., Verdugo, M., \& Gómez, J. (2011). Escala de calidad de vida familiar para familias de personas con discapacidad. Manual. Adaptación para Colombia. Salamanca, ES: Universidad del Salamanca.

Farran, L.K., Lederberg, A.R., Jackson, L.A. (2009). Maternal input and lexical development: The case of deaf preschoolers. International Journal of Language Eु Communication Disorders, 44, 145-163 doi:10.1080/13682820801973404

Fernández-Faúndez, E., Arias, B., Gómez, L., \& Jorrín, I. (2012). Calidad de vida en familias de personas mayores con discapacidad intelectual. Siglo Cero, 43(4), 31-48.

Fornell, C., \& Larcker, D. (1981). Evaluating structural equation models with unobservable variables and measurement error. Journal of Marketing Research, 18(1), $39-50$.

Giné, C., Gràcia, M., Vilaseca, R., Beltran, F., Balcells, A., Dalmau, M., ... Mas, J. (2015). Family quality of life for people with intellectual disabilities in Catalonia. Journal of Policy and Practice in Intellectual Disabilities, 12(4), 244-254. https://doi.org/10.1111/jppi.12134

Giné, C., Vilaseca, R., Gràcia, M., Mora, J., Orcasitas, J. Ç., Simón, C., ... Simó-Pinatella, D. (2013). 
Spanish Family Quality of Life Scales: under and over 18 years old. Journal of Intellectual and Developmental Disability, 38(2), 141-148. https:// doi.org/10.3109/13668250.2013.774324

Goldbeck, L., Melches, J., Franz, A., Vossbeck, S., Lang, D., \& Mihatsch, W. (2005). Quality of life in families with chronic pediatric heart diseases. Kindheit und Entwicklung, 14(2), 79-86. https:// doi.org/10.1026/0942-5403.14.2.79

González-del-Yerro, A., Simón, C., Cagigal, V., \& Blas, E. (2013). La calidad de vida de las familias de personas con discapacidad intelectual. Un estudio cualitativo realizado en la comunidad de Madrid. Revista Española de Orientación y Psicopedagogía, 24(1), 93-109.

Hair, J., Anderson, R., Tatham, R., \& Black, W. (2009). Multivariate data analysis (C. University Ed.). Upper Saddle River: Prentice-Hall.

Hoffman, L., Marquis, J., Poston, D., Summers, J., \& Turnbull, A. (2006). Assessing family outcomes: Psychometric evaluation of the Beach Center Family Quality of Life Scale. Journal of Marriage and Family, 68(4), 1069-1083. https://doi.org/10.1111/ j.1741-3737.2006.00314.x

Loga, S., Sosic, B., Kulenovic, A., Svraka, E., Bosankic, N., Kucukalic, A., ... Hadzic, A. (2012). Chronic illness and family: impact of schizophrenia and crohn's disease on the family quality of life. Psychiatria Danubina, 24(4), 359-366.

Muñoz, Y., Poblete, Y., \& Jiménez, A. (2012). Calidad de vida familiar y bienestar subjetivo en jóvenes con discapacidad intelectual de un establecimiento con educación especial y laboral de la ciudad de Talca. Interdisciplinaria, 29(2), 207-221.

Navarrete, L., \& Ossa, C. (2013). Estilos parentales y calidad de vida familiar en adolescentes con conductas disruptivas. Ciencias Psicológicas, 7(1), 47-56.

Ossa, C., Navarrete, L., \& Jiménez, A. (2014). Estilos parentales y calidad de vida familiar en padres y madres de adolescentes de un establecimiento educacional de la ciudad de Chillán (Chile). Investigación $\mathcal{B}$ Desarrollo, 22(1), 19-37.

Park, J., Hoffman, L., Marquis, J., Turnbull, A. P., Poston, D., Mannan, H., ... Nelson, L. L. (2003). Toward assessing family outcomes of service delivery: Validation of a family quality of life survey. Journal of Intellectual Disabilty Research, 47(4), 367-384. https:// doi.org/10.1046/j.1365-2788.2003.00497.x

Rodríguez, A., Verdugo, M., \& Sánchez, M. (2008). Calidad de vida familiar y apoyos para los progenitores de personas con discapacidad intelectual en proceso de envejecimiento. Siglo Cero, 39(3), 19-34.

Sainz, F., \& Verdugo, M. A. (2006). Adaptación de la Escala de Calidad de Vida Familiar al contexto español. En M. A. Verdugo (Ed.), Como mejorar la calidad de vida de personas con discapacidad (pp. 299-322). Salamanca: Amarú.

Samuel, P., Rillota, F., \& Brown, I. (2012). The development of family quality of life concepts and measures. Journal of Intellectual Disability Research, 56(1), 1-16. https://doi.org/10.1111/ j.1365-2788.2011.01486.x

Schalock, R., \& Verdugo, M. (2007). El concepto de calidad de vida en los servicios y apoyos para personas con discapacidad intelectual. Siglo Cero, 38(4), 21-36.

Verdugo, M. A., Córdoba, L., \& Gómez, J. (2005). Spanish adaptation and validation of the Family Quality of Life Survey. Journal of Intellectual Disability Research, 49, 794-798. https:// doi.org/10.1111/j.1365-2788.2005.00754.x

Wang, M. (2010). Quality of life of individuals with intellectual disabilities and their families in China: Research and applications. En R. Kober (Ed.), Enhancing the quality of life of people with intellectual disabilities (Vol. 41, pp. 163-172). Nueva York: Springer.

Zuna, N., Turnbull, A., \& Summers, J. (2009). Family Quality of Life: Moving from measurement to application. Journal of Policy and Practice in 
Natalia Bello-Escamilla, Joucelyn Rivadeneira, Marcela Concha-Toro, Et al.

Intellectual Disabilities, 6(1), 25-31. https:// doi.org/10.1111/j.1741-1130.2008.00199.x

\section{Notas}

* Artículo de investigación. 